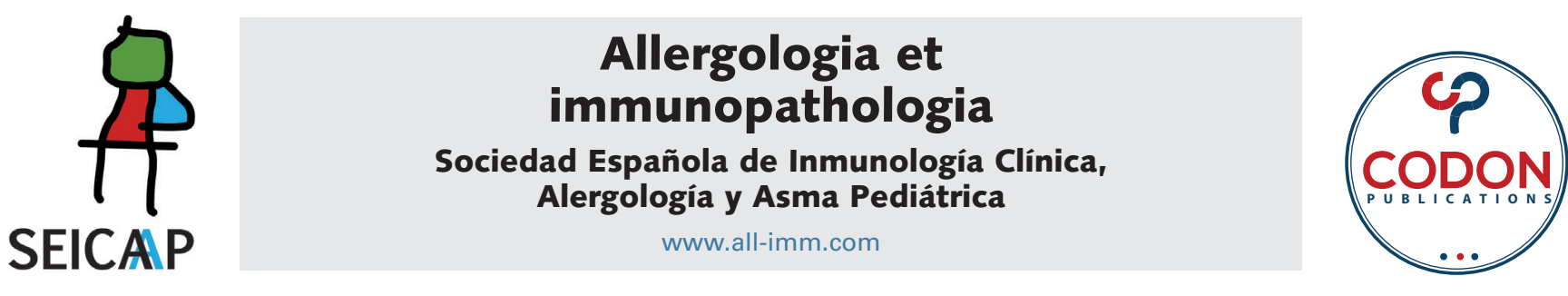

\title{
Pneumococcal vaccine in patients with idiopathic juvenile arthritis in treatment with tumor necrosis factor inhibitors
}

\author{
Javiera Berho ${ }^{a}$, María-Paulina Monsalvez ${ }^{a}$, Jorge Rojas ${ }^{a}$, Jaime Inostroza ${ }^{b}$, \\ Arnoldo Quezada ${ }^{\mathrm{a}, \mathrm{c} *}$
}

${ }^{a}$ Department of Paediatrics, School of Medicine, University of Chile, Santiago de Chile, Chile

${ }^{b}$ Clinical Laboratory, University of La Frontera, Jeffrey Modell Centre, Temuco, Chile

'Paediatric Rheumatology International Trials Organization (PRINTO), Genova, Italy

Received 13 October 2020; Accepted 9 November 2020

Available online 1 July 2021

\section{KEYWORDS \\ adalimumab; \\ etanercept; \\ immunosuppressive \\ drugs; \\ juvenile idiopathic \\ arthritis; \\ monoclonal \\ antibodies; \\ pneumococcal \\ vaccine}

\begin{abstract}
Introduction: Juvenile idiopathic arthritis (JIA) is the most common rheumatological disease of childhood. The therapy with tumor necrosis factor (TNF) inhibitors (TNFi) in JIA patients has demonstrated efficacy and safety. The most reported adverse event is the high susceptibility to infections. Preventive vaccination helps to decrease these risks. The information on response to vaccines in JIA patients having treatment with anti-TNF is limited.

Objectives: To evaluate the response to pneumococcal vaccine in JIA patients undergoing treatment with mAb.

Materials and methods: Analytical observational mixed cohort study. Data obtained from the clinical records of an immunorheumatology polyclinic of a metropolitan hospital in Santiago (Chile). Treatments, pneumococcal vaccine schedules, immunological laboratory, and measurement of specific antibodies against 10 pneumococcal serotypes were recorded.

Results: Nineteen patients were included; average age was 13.8 years; and average evolution time of the disease was 46.2 months. Adalimumab (Humira $\left.{ }^{\circledR}\right)$ was indicated in case of 13 patients $(68.4 \%)$ and etanercept $\left(\right.$ Enbrel $\left.^{\circledR}\right)$ to $6(31.5 \%)$. The most indicated scheme was a dose of 13-valent pneumococcal conjugate vaccine (PCV13) followed at 8 weeks by a dose of pneumococcal polysaccharide vaccine (PPSV23) in nine (47.3\%) patients. Seventeen (89.4\%) patients were on immunosuppressive treatment at the time of vaccination. Only one patient did not meet the criteria for response to vaccine.

Conclusions: The pneumococcal vaccine induces protective levels of serum antibodies in JIA patients undergoing TNFi treatment. The vaccination schedule and the lymphocyte count could influence the response capacity.
\end{abstract}

(c) 2021 Codon Publications. Published by Codon Publications.

${ }^{*}$ Corresponding author: Arnoldo Quezada. Department of Paediatrics, School of Medicine, University of Chile, Santiago de Chile, Chile. E-mail address: aquezada@med.uchile.cl 


\section{Introduction}

Juvenile idiopathic arthritis (JIA) is the most prevalent rheumatological disease of childhood. ${ }^{1}$ Genetic and environmental factors are involved in the pathogenesis of this heterogeneous and multifactorial autoimmune disease. ${ }^{2}$ The International League of Associations for Rheumatology (ILAR) has defined JIA subtypes on the basis of clinical characteristics during the first 6 months of the disease, the number of affected joints, the presence of extraarticular manifestations, and rheumatoid factor (RF). The current classification includes seven subgroups (systemic, oligoarticular, polyarticular with negative or positive RF, associated with enthesitis, associated with psoriasis, and undifferentiated arthritis). ${ }^{3}$ The treatment plan for JIA includes education of the patient, their family, and their school environment, pharmacological treatment with non-steroidal anti-inflammatory drugs (NSAIDs), glucocorticoids (intra-articular and systemic), and disease-modifying antirheumatic drugs (DMARDs). In the event of failure, the use of monoclonal antibodies (mAbs) is considered. ${ }^{4}$

The mAbs against some cytokines or their receptors have been used to restore, stimulate, or reduce the immune response of the host, and its use in JIA has shown efficacy and safety, has reduced morbidity, and improved the quality of life of these patients. Although mAbs can modify the course of JIA interacting with the host's immune system and altering its response capacity, they are also capable of inducing immunosuppression. ${ }^{5}$ By themselves or in conjunction with the concomitant use of other immunosuppressive agents, such as glucocorticoids and methotrexate (MTX), together with the underlying inflammatory disease, they may contribute to an increased risk of infections. ${ }^{6}$ Infections associated with the use of mAbs have been studied widely, concluding that there is an increased risk of viral and bacterial infections, especially by mycobacteria, with the use of tumor necrosis factor-alpha (TNF-a) inhibitors (TNFi). Less evidence exists for the increased risk of fungal infections and other intracellular microorganisms. ${ }^{7,8}$ Lymphopenia less than $600 / \mathrm{mL}$, even more less than 250 CD4 T lymphocytes (LT)/mL, and treatment with glucocorticoids in doses of more than $10 \mathrm{mg}$ of prednisolone or its equivalent per day, are more frequently associated with infections. ${ }^{9}$ JIA patients, because of immunosuppression, are at increased risk of reactivation of tuberculosis, chickenpox, and infections by capsulated bacteria. ${ }^{10}$ Preventive vaccination helps to decrease these aforementioned risks. ${ }^{11}$

Various publications have demonstrated the safety and efficacy of different vaccines in patients with rheumatological diseases, in spite of the use of immunosuppressants..$^{12-15}$ However, vaccination in special situations is an undervalued issue. ${ }^{13,14}$ Vaccines may not induce adequate immune response, especially the pneumococcal and influenza vaccines, in patients with drug immunosuppression. ${ }^{14,16}$ In paediatric cases the investigation is much less, ${ }^{17,18}$ and the information on response to vaccines in patients with JIA having TNFi treatment is limited.

Streptococcus pneumoniae (pneumococcus) has a capsule composed of a complex of polysaccharides that represents its main virulence factor and interferes with phagocytosis of neutrophils and macrophages. The highest incidence of invasive pneumococcal disease occurs in children aged less than 2 years and are in risk groups, in which are immunosuppressed patients. ${ }^{19,20}$ Available pneumococcal vaccines include polysaccharides and conjugates. Among the pneumococcal polysaccharide vaccines, Pneumococcal PolySaccharide Vaccine 23 valent (PPSV23) is an inactivated vaccine which includes 23 serotypes. PPSV23 vaccines have been shown to be effective in the prevention of invasive pneumococcal disease; however, their efficacy is lower in immunosuppressed patients. ${ }^{14,21}$ Among the pneumococcal conjugate vaccines (PCV), PCV10 and PCV13 are available. There are different vaccination schedules against pneumococcus recommended by the Centers for Disease Control and Prevention (CDC) for children having immunosuppressive treatment. ${ }^{22-24}$ Evaluation of response to vaccines is not a routine practice in patients receiving immunosuppressive therapy.

The objective of this study was to evaluate response to pneumococcal vaccine in patients with JIA undergoing mAb treatment.

\section{Methods}

It was an analytical observational mixed cohort study, with data obtained from the clinical records of the immunorheumatology out-patients unit of the Exequiel González Cortés Hospital, Santiago de Chile, Chile. All registered JIA patients with an indication of $\mathrm{mAb}$, who had received the available pneumococcal vaccine and who gave their informed consent, authorized by their legal guardian, were included in the study. Those patients who did not attend medical controls, or who had been transferred to another health center, or who did not give or withdraw their informed consent were excluded. To assess response to the vaccine, specific immunoglobulin $\mathrm{G}(\mathrm{lgG})$ antibodies $(\mu \mathrm{g} / \mathrm{mL})$ against 10 pneumococcal serotypes $(1,3,4,5,6 \mathrm{~B}, 9 \mathrm{~V}, 14,18 \mathrm{C}, 19$, and $23 \mathrm{~F}$ ) were measured by the ELISA method. Subjects who had values higher than or equal to $1.3 \mu \mathrm{g} / \mathrm{mL}$ in $50 \%$ or more of the serotypes in children aged less than 6 years, and $70 \%$ or more of the serotypes in subjects aged 6 years or older were considered "responders", otherwise they were classified as "non-responders". In addition, the number of responding serotypes was recorded, with a value greater than or equal to $1.3 \mu \mathrm{g} / \mathrm{mL}$ for each of the patients, and the responder serotype was defined as obtaining a value greater than or equal to $1.3 \mu \mathrm{g} / \mathrm{mL}$ for each of the serotypes. ${ }^{25}$ Simultaneously, a complete blood count, immunoglobulins count, and complement were performed. Concomitant administration of methotrexate, systemic corticosteroids, and other immunosuppressive treatments was recorded.

Informed consent was obtained from the legal guardian of each patient, and the study was approved by the ethics committee of the local health service.

\section{Statistical analysis}

The variables were treated using Stata 13. For the dichotomous variables, the proportions were calculated over the total sample. For the nominal and ordinal variables, the proportions were calculated on the valid observations for 
each variable, which corresponded to the sum of the frequencies of each category of the variable.

Three types of correlation analysis were performed: between categorical variables, between continuous variables, and between categorical and continuous variables. Only bivariate analyses were applied due to the small sample size. Pearson's $\chi^{2}$ test was applied to test the independence between categorical variables. The relationship between continuous variables was measured using Pearson's correlation coefficient and a hypothesis test was applied on this parameter to each measurement. To analyze the relationship between continuous and dichotomous variables, the biserial point relationship was measured, considering a confidence level of $95 \%$. A correlation analysis of variables was carried out to determine whether the demographics of the patients, the characteristics of the baseline JIA, the treatment, the vaccination schedules, and/or the laboratory could have influenced the response of specific antibodies against pneumococcus. The sample size only allowed bivariate analyses, and it was not possible to apply a multivariate regression model.

\section{Results}

Twenty five patients controlled for JIA undergoing treatment with mAb were registered; four were excluded because they did not attend controls and two were excluded due to transfer to other health centers, and finally 19 patients were included for the analysis, with an average age of 13.8 years, and an average time of evolution of the disease being 46.2 months. Adalimumab (Humira ${ }^{\circledR}$ ) was indicated in 13 cases (68.5\%) and etanercept (Enbrel ${ }^{\circledR}$ ) to six $(31.5 \%)$ patients. Before starting the mAb, the pneumococcal vaccine was indicated. The most indicated scheme was a dose of 13-valent pneumococcal conjugate vaccine (PCV13, Prevenar-13 ${ }^{\circledR}$ ) followed at 8 weeks by a dose of pneumococcal polysaccharide vaccine (PPSV23, Pneumo-23 ${ }^{\circledast}$ ) to nine $(47.3 \%)$ patients. A single dose of PPSV23 was administered to eight (42.2\%) patients and PCV13 to two (10.5\%) others. The mean time between the administration of the last dose of pneumococcal vaccine and the start of mAb was 3 months.

The leukocyte, lymphocyte, immunoglobulin, and complement counts were normal in all patients.

Seventeen (89.4\%) patients were on immunosuppressive treatment at the time of vaccination. Sixteen $(84.2 \%)$ received methotrexate (MTX), eight $(42.1 \%)$ received prednisone, and seven (41.1\%) of the 17 patients having MTX used prednisone concomitantly. No use of systemic glucocorticoids was recorded at the time of vaccination. Only one patient used other immunosuppressants, specifically sulfasalazine associated with azathioprine.

At the time of evaluation of the response to vaccination, all patients were receiving some immunosuppressive treatment, in addition to mAb. Eighteen (94.7\%) received MTX and 10 (52.6\%) received systemic glucocorticoids, including prednisone and methylprednisolone. Nine (50\%) of the 18 MTX patients were concomitantly using systemic glucocorticoids.

Only one patient did not meet the criteria for responders to the vaccine, adjusted for age, corresponding to a female patient, without other comorbidities, with a polyarticular RF positive JIA undergoing treatment with MTX and systemic glucocorticoids at the time of single-dose PPSV23 vaccination. The study by serotypes showed a lower percentage of patients responding to serotypes 1 (S1) $(\mathrm{n}=$ $12,63.1 \%)$, S3 ( $n=14,73.6 \%)$, and S4 ( $=13,68.4 \%) ; 18$ responded to other serotypes (94.7\%) (Table 1$)$.

A significant correlation of the vaccination scheme with specific antibodies against serotype 5 (S5) $(P=0.011)$ was observed, highlighting that a single dose of PCV13 was the only scheme that presented cases without response to serotype 5, while the scheme of PCV13 followed by PPSV23 was the one that showed the highest number of responses (Table 2).

A significant correlation close to $5 \%$ was also identified between the lymphocyte count and the specific antibodies against serotype $4(P=0.054)$, showing a lower average number of lymphocytes $(2344 / \mu \mathrm{L})$ in non-responders, compared to responders $(3535 / \mu \mathrm{L})$, although not constituting lymphopenia. In spite of its lower level of significance, this correlation fulfilled all the assumptions of the applied tests as well as normal distribution and required variance.

Table 1 Results of specific antibodies against pneumococcus.

\begin{tabular}{lcc}
\hline & $\begin{array}{c}\text { Responders } \\
\mathrm{n}(\%)\end{array}$ & $\begin{array}{c}\text { Non-responders } \\
\mathrm{n}(\%)\end{array}$ \\
\hline $\begin{array}{l}\text { Response to pneumococcal } \\
\text { vaccine adjusted for age* }\end{array}$ & $18(94.7)$ & $1(5.2)$ \\
Serotype 1 & $12(63.1)$ & $7(36.8)$ \\
Serotype 3 & $14(73.6)$ & $5(26.3)$ \\
Serotype 4 & $13(68.4)$ & $6(31.5)$ \\
Serotype 5 & $18(94.7)$ & $1(5.2)$ \\
Serotype 6B & $18(94.7)$ & $1(5.2)$ \\
Serotype 9V & $17(89.4)$ & $2(10.5)$ \\
Serotype 14 & $19(100)$ & $0(0)$ \\
Serotype 18C & $18(94.7)$ & $1(5.2)$ \\
Serotype 19F & $19(100)$ & $0(0)$ \\
Serotype 23F & $18(94.7)$ & $1(5.2)$ \\
\hline
\end{tabular}

*Response to age-adjusted pneumococcal vaccine: $\geq 1.3 \mu \mathrm{g} / \mathrm{mL}$ in $\geq 50 \%$ of serotypes in patients aged $<6$ years and $\geq 70 \%$ of serotypes in patients aged $\geq 6$ years.

Table 2 Response of specific antibodies against serotype 5 (S5) according to the pneumococcal vaccination scheme.

\begin{tabular}{lccc}
\hline $\begin{array}{l}\text { Pneumococcal } \\
\text { vaccination scheme }\end{array}$ & Responders & Non-responders & Total \\
\hline PCV13 + PPSV23 & 9 & 0 & 9 \\
PPSV23 & 8 & 0 & 8 \\
PCV13 & 1 & 1 & 2 \\
TOTAL & 18 & 1 & 19 \\
\hline
\end{tabular}

Pearson $\chi^{2}=8.9722 ; \mathrm{P}=0.011$. 


\section{Discussion}

TNFi is the treatment of choice in refractory oligoarticular and polyarticular JIA, and for early use in arthritis with enthesitis, etanercept being the first line of treatment, followed by adalimumab and infliximab, although the latter is used less widely. ${ }^{26,27}$ In our patients, adalimumab followed by etanercept was mainly indicated, and in case of failure it was decided to switch to the second TNFi with a good response.

In a systematic review published by Van Aalst et al., ${ }^{16}$ it was concluded that in patients treated with immunosuppressants, the response to PCV and PPSV vaccines is altered, compared to controls, and that this response is lower for PCV than PPSV. When comparing patients treated with immunosuppressants versus those treated with TNFi, it was observed that the latter group had a more favorable response to vaccines. ${ }^{16}$

In paediatric patients, research is limited. In a study published by Aikawa et al. ${ }^{28}$ that included 17 JIA patients treated with TNFi (etanercept) in association with MTX, compared to $10 \mathrm{JIA}$ patients with stable doses of MTX, both groups received a dose of PPV23, it was concluded that the therapy with TNFi did not appear to have an additional detrimental effect on the short- and long-term compared to MTX alone. In another study published by Farmaki et al., ${ }^{17}$ 63 children with different types of JIA were studied, 31 children received TNFi (etanercept or adalimumab) and MTX or cyclosporine with or without prednisone, and the control group of 32 children received only MTX or cyclosporine or a combination of both, with or without prednisone. All subjects were vaccinated with PCV7. On comparing the response in both groups, all participants achieved optimal levels of seroconversion; however, the group with TNFi reached lower levels than the control group. ${ }^{17}$ Current CDC recommendations indicate that patients aged 6-18 years on immunosuppressive treatment who have not received pneumococcal vaccine are administered one dose of PCV13, and one dose of PPSV23 is administered at 8 weeks after the PCV13 dose. ${ }^{24}$ It is suggested that the second dose of PPSV23 is administered at less than 5 years of its first dose, but other studies consider this second dose as unnecessary. ${ }^{29}$ Most of our patients received another regimen-either a single dose of PPSV23 or PCV13 due to its availability in the national public health system at the time of starting the mAb. Correlation analysis showed a favorable response with the different schemes used, except a statistically significant lower response to serotype 5 in case of vaccination with a single dose of PCV13.

The evaluation of the response to the pneumococcal vaccine in Chile is carried out by measuring IgG antibodies against 10 pneumococcal serotypes, and it is based on the percentage of serotypes that reach levels considered as protective. We evidenced $63-73 \%$ of patients responding to serotypes 1,3 , and 4 , which is lower when compared to the rest of the serotypes in which more than $94 \%$ of patients responded. Farmaki et al. reported a significantly lower seroconversion against serotypes 4, 14, and $23 \mathrm{~F}$ in patients with JIA treated with TNFi associated with DMARD, when compared with the control group treated only with DMARD. ${ }^{17}$ If TNFi affect vaccine response, the literature is controversial. $12,14,16,17,21$ In our study, the MTX and
TNFi treatments did not significantly alter response to the vaccine.

Alterations in the immune system associated with the use of immunosuppressants could affect response to vaccines. ${ }^{15,22}$ In the present study, the use of MTX showed no difference in response to the vaccine. In parallel, the increased risk of infections associated with the use of glucocorticoids has been demonstrated widely, especially in lymphopenic patients, but there are no data regarding its role in response to pneumococcal vaccines. ${ }^{10,12}$

Patients receiving chronic immunosuppressive therapy with glucocorticoids and/or DMARDs can develop severe lymphopenia, involving all types of lymphocytes. A lymphopenia less than $600 / \mathrm{mL}$, but even less than $250 \mathrm{CD} 4 \mathrm{LT} / \mathrm{mL}$ and treatment with glucocorticoids in doses of more than $10 \mathrm{mg}$ of prednisolone or its equivalent per day are more frequently associated with infections..$^{10,22}$

The lower lymphocyte count with a correlation of significance close to $5 \%$ and the response of specific antibodies to serotype 4 among non-responders in our study make it necessary to directly corroborate and/or assess response to pneumococcal vaccine in lymphopenic patients or those with low lymphocyte count below the cut-off point.

In order to avoid adverse effects of live vaccines and to obtain an optimal response from attenuated vaccines, it is recommended to administer them in the initial stages of the disease, when the degree of immunosuppression is minimal. $5,12,30$

Several publications have demonstrated the safety and efficacy of inactivated vaccines and protein components, such as hepatitis A and B, influenza, pneumococcus, meningococcus, haemophilus, poliomyelitis, and tetanus, in patients with rheumatological diseases, in spite of the use of immunosuppressants. However, it is of great importance to know that vaccines may not induce adequate immune response, especially the pneumococcal and influenza vaccines, in patients with pharmacological immunosuppression. ${ }^{12,15}$

The variability in response to the vaccine evidenced in our study could be attributed to several factors, including different vaccination schedules used due to their availability in the public health system, and the use of immunosuppressants at the time of vaccination.

A limitation of the study was its small sample size, which prevented the performance of a multivariate logistic regression.

\section{Conclusions}

The pneumococcal vaccine is indicated in JIA patients prior to the initiation of mAb. Currently, the response to immunizations is not routinely observed in this subgroup of patients. The evaluation of this response could provide valuable information to identify factors that are associated with seroconversion, to optimize vaccination schedules, and to improve protection against infections in more susceptible patients.

The present study showed that the antibody response conferred by the pneumococcal vaccine was satisfactory in JIA patients undergoing TNFi treatment, and identified some variables that could influence their response. The 
pneumococcal vaccine induced protective levels of serum antibodies in these JIA patients treated with TNFi. The vaccination schedule and the lymphocyte count could influence the response capacity.

\section{Conflict of interest}

The authors declare no conflicts of interest.

\section{Funding}

No specific funding was received to carry out this study.

\section{References}

1. Prakken B, Albani S, Martini A. Juvenile idiopathic arthritis. Lancet. 2011;377: 2138-49. https://doi.org/10.1016/S01406736(11)60244-4

2. Palman J, Shoop-Worrall S, Hyrich K, MCDonagh JE. Update on the epidemiology, risk factors and disease outcomes of juvenile idiopathic arthritis. Best Pract Res Clin Rheumatol. 2018 Apr;32(2):206-222. https://doi.org/10.1016/j. berh.2018.10.004

3. Petty RE, Southwood TR, Manners P, Baum J, Glass DN, Goldenberg $\mathrm{J}$ et al. International league of associations for rheumatology classification of juvenile idiopathic arthritis: Second revision, Edmonton, 2001. J Rheumatol. 2004 Feb;31(2):390-2. PMID: 14760812.

4. Blazina S, Markelj G, Avramovic MZ, Toplak N, Avcin T. Management of juvenile idiopathic arthritis: A clinical guide. Pediatr Drugs. 2016;18:397-412. https://doi.org/10.1007/ s40272-016-0186-0

5. Vanoni F, Minoia F, Malattia C. Biologics in juvenile idiopathic arthritis: A narrative review. Eur J Pediatr. 2017;176:1147-53. https://doi.org/10.1007/s00431-017-2960-6

6. Davies HD, AAP Committee on Infectious Diseases. Infectious complications with the use of biologic response modifiers in infants and children. Pediatrics. 2016;138(2):e20161209. https://doi.org/10.1542/peds.2016-1209

7. Diener C, Horneff G. Comparison of adverse events of biologicals for treatment of juvenile idiopathic arthritis: A systematic review. Expert Opin Drug Saf. 2019;18:8719-32. https:// doi.org/10.1080/14740338.2019.1632288

8. Lee WJ, Lee TA, Suda KJ, Calip GS, Briars L, Schumock GT. Risk of serious bacterial infection associated with tumour necrosis factor-alpha inhibitors in children with juvenile idiopathic arthritis. Rheumatology. 2018;57:273-82. https://doi. org/10.1093/rheumatology/kex049

9. Gluck T, Kiefmann B, Grohmann M, Falk W, Straub RH, Scholmerich J. Immune status and risk for infection in patients receiving chronic immunosuppressive therapy. J Rheumatol. 2005;32:1473-80. PMID: 16078322

10. Nagy A, Mátrai P, Hegyi P, Alizadeh H, Bajor J, Czopf L et al. The effects of TNF-alpha inhibitor therapy on the incidence of infection in JIA children: A meta-analysis. Ped Rheumatol. 2019;17:4. https://doi.org/10.1186/s12969-019-0305-x

11. Heijstek MW, Ott de Bruin LM, Bijl M, Borrow R, van der Klis F, Koné-Paut $I$ et al. EULAR recommendations for vaccination in paediatric patients with rheumatic diseases. Ann Rheum Dis. 2011;70:1704-12. https://doi.org/10.1136/ard.2011.150193

12. Conti F, Rezai S, Valesini G. Vaccination and autoimmune rheumatic diseases. Autoimm Rev. 2008;8(2):124-8. https:// doi.org/10.1016/j.autrev.2008.07.007
13. Morin MP, Quach C, Fortin E, Chédeville G. Vaccination coverage in children with juvenile idiopathic arthritis followed at a paediatric tertiary care centre. Rheumatology (Oxford). 2012 Nov;51(11):2046-50. https://doi.org/10.1093/ rheumatology/kes175

14. Sousa S, Duarte AC, Cordeiro I, Ferreira J, Gonçalves MJ, Meirinhos T et al. Efficacy and safety of vaccination in pediatric patients with systemic inflammatory rheumatic diseases: A systematic review of the literature. Acta Reumatol Port. 2017;42:8-16.

15. Martire B, Azzari Ch, Badolato R, Canessa C, Cirillo E, Gallo $\mathrm{V}$ et al. Vaccination in immunocompromised host: Recommendations of Italian Primary Immunodeficiency Network Centers (IPINET). Vaccine. 2018;36:3541-54. https:// doi.org/10.1016/j.vaccine.2018.01.061

16. van Aalst M, Langedijk AC, Spijker R, de Bree GJ, Grobusch MP, Goorhuis A. The effect of immunosuppressive agents on immunogenicity of pneumococcal vaccination: A systematic review and meta-analysis. Vaccine. 2018 Sep 18;36(39):583245. https://doi.org/10.1016/j.vaccine.2018.07.039

17. Farmaki E, Kanakoudi-Tsakalidou F, Spoulou V, Trachana M, Pratsidou-Gertsi P, Tritsoni $M$ et al. The effect of anti-TNF treatment on the immunogenicity and safety of the 7-valent conjugate pneumococcal vaccine in children with juvenile idiopathic arthritis. Vaccine 2010;28:5109-13. https://doi. org/10.1016/j.vaccine.2010.03.080

18. Lipsitch M, Li LM, Patterson S, Trammel J, Juergens C, Gruber WC, et al. Serotype-specific immune responses to pneumococcal conjugate vaccine among children are significantly correlated by individual: Analysis of randomized controlled trial data. Vaccine. 2018 Jan 25;36(4):473-8. https:// doi.org/10.1016/j.vaccine.2017.12.015

19. van der Poll T, Opal SM. Pathogenesis, treatment, and prevention of pneumococcal pneumonia. Lancet. 2009 Oct 31;374(9700):1543-56. PMID: 19880020. https://doi. org/10.1016/S0140-6736(09)61114-4

20. Vernatter J, Pirofski LA. Current concepts in host-microbe interaction leading to pneumococcal pneumonia. Curr Opin Infect Dis. 2013 Jun;26(3):277-83. https://doi.org/10.1097/ QCO.0b013e3283608419

21. Kuronuma K, Takahashi H. Immunogenicity of pneumococcal vaccines in comorbid autoimmune and chronic respiratory diseases. Hum Vaccin Immunother. 2019;15(4):859-62. https:// doi.org/10.1080/21645515.2018.1564443

22. Centers for Disease Control and Prevention. Use of 13-valent pneumococcal conjugate vaccine and 23-valent pneumococcal polysaccharide vaccine among children aged 6-18 years with immunocompromising conditions: Recommendations of the Advisory Committee on Immunization Practices (ACIP). MMWR Morb Mortal Wkly Rep. 2013;62(25):521-4.

23. American Academy of Pediatrics, Committee on Infectious Diseases. Policy statement-Immunization for streptococcus pneumoniae infections in high-risk children. Pediatrics. 2015 May;135(5):945. https://doi.org/10.1542/peds.2015-0451

24. Centers for Disease Control and Prevention. Recommended immunization schedules for persons aged 0 through 18 years. CDC 2019 [Internet]. Available from: https://www.cdc.gov/ vaccines/schedules/downloads/past/2019.pdf Accessed on October 13, 2020.

25. Quezada A, Maggi L, Norambuena X, Inostroza J, Quevedo F. Response to pneumococcal polysaccharide vaccine in children with asthma, and children with recurrent respiratory infections, and healthy children. Allergol Immunopathol. 2016;44(4):376-81. https://doi.org/10.1016/j.aller.2016.01.003

26. Taddio A, Cattalini C, Simonini G, Cimaz R. Recent advances in the use of anti-TNFa therapy for the treatment of juvenile idiopathic arthritis. Expert Rev Clin Immunol. 2016;12(6):64149. https://doi.org/10.1586/1744666X.2016.1146132 
27. Cimaz R, Marino A, Martini A. How I treat juvenile idiopathic arthritis: A state of the art review. Autoimmun Rev. 2017;16:1008-1015. https://doi.org/10.1016/j.autrev.2017.07.014

28. Aikawa NE, França ILA, Ribeiro AC, Sallum AME, Bonfa E, Silva CA. Short- and long-term immunogenicity and safety following the 23-valent polysaccharide pneumococcal vaccine in juvenile idiopathic arthritis patients under conventional DMARDs with or without anti-TNF therapy. Vaccine 2015;33:604-9. https://doi.org/10.1016/j.vaccine.2014.12.030
29. Broyde A, Arad U, Madar-Balakirski N, Paran D, Kaufman I, Levartovsky $D$ et al. Long-term efficacy of an antipneumococcal polysaccharide vaccine among patients with autoimmune inflammatory rheumatic diseases. J Rheumatol. 2016 Feb;43(2):267-72. https://doi.org/10.3899/jrheum.150397

30. Rakoczi E, Szekanecz Z. Pneumococcal vaccination in autoimmune rheumatic diseases. RMD Open. 2017;3:e000484. https://doi.org/10.1136/rmdopen-2017-000484 